\title{
AVALIAÇÃO NÃO DESTRUTIVA DE ESTRUTURAS DE MADEIRAS EM EDIFÍCIO HISTÓRICO DE TIRADENTES, MG
}

\author{
Luciana Barbosa de Abreu*, José Tarcísio Lima', Giovanni Francisco Rabelo', \\ Francisco Carlos Gomes ${ }^{1}$, Paulo Fernando Trugilho ${ }^{1}$, Felipe de Souza Eloy ${ }^{1}$
}

*Autora para correspondência: luciana@deg.ufla.br

\begin{abstract}
RESUMO: Problemas relacionados à durabilidade da madeira são comumente encontrados em estruturas de edificações históricas. Medidas de preservação e conservação devem ser adotadas, a fim de se evitar perdas e substituições que descaracterizam as construções. Métodos não destrutivos para a detecção de deteriorações podem ser utilizados, a fim de embasar decisões e aumentar a longevidade do patrimônio histórico. Nesse contexto, este trabalho foi realizado com o objetivo de realizar ensaios não destrutivos para inferir sobre a integridade de uma viga e um pilar originais da construção do Sobrado Ramalho, edifício histórico de Tiradentes, MG. Foram utilizados os equipamentos stress wave timer e resistógrafo. Amostras das peças foram retiradas para análises de densidade. Os resultados mostraram que, em ambas as estruturas, para cálculo dos módulos de elasticidade dinâmicos, não houve diferença significativa na aplicação do stress wave timer nos alinhamentos estudados. Não houve diferença significativa entre as direções de aplicação do resistógrafo no pilar, por ele ser aparentemente íntegro, de seções regulares, praticamente quadradas, e por não sofrer carregamento excêntrico. Já na viga houve diferença significativa, supostamente pelo fato de ela possuir trincas na sua linha de tração. Os equipamentos, pouco conhecidos no meio técnico conservacionista de patrimônio, permitem metodologias promissoras para inspeção de estruturas de madeira em serviço.
\end{abstract}

Palavras-chave: Avaliação não destrutiva, estrutura de patrimônio histórico, deterioração, integridade.

\section{NON-DESTRUCTIVE EVALUATION OF TIMBER STRUCTURES IN A HISTORICAL BUILDING OF TIRADENTES, MG}

\begin{abstract}
Problems related to the durability of wood are commonly found in historical buildings structures. Preservation and conservation resolutions must be adopted, in order to avoid losses and substitutions, which mischaracterize buildings. Non-destructive methods for detecting deterioration should be used in order to substantiate decisions and increase the longevity of historical heritage. This work was carried out in order to perform non-destructive essays to infer about the integrity of a beam and a pillar of the original construction of the Sobrado Ramalho, a historical building of the city of Tiradentes, MG. The equipments utilized were the Stress Wave Timer and resistograph. Samples of the elements were taken for analysis of density. The results showed that, in both structures, to calculate the dynamic modulus of elasticity, there was no significant difference for the application of stress wave timer on the alignments studied. There was no significant difference between the directions of application of the resistograph on the pillar, due to its apparent entirety and regular sessions, practically square, and to not being loaded eccentrically. In the case of the beam, there was significant difference, presumably because it has cracks in its traction line. The equipments, unknown by professionals of heritage conservation allow promising methodologies for inspection of timber structures in service.
\end{abstract}

Key words: Non destructive essay, historical heritage timber structure, deterioration, entirety.

\section{INTRODUÇÃO}

É notória a utilização de madeira nas edificações históricas brasileiras. Entretanto, problemas relacionados à durabilidade da madeira são comumente encontrados, uma vez que nenhuma espécie de madeira, nem mesmo aquelas de reconhecida durabilidade natural, é capaz de resistir, indefinidamente, às variações das condições ambientais, ao ataque de microrganismos xilófagos e ao excesso de cargas. $\mathrm{O}$ respeito ao patrimônio e o entendimento de seu valor estético, histórico e social preconizam a necessidade de sua preservação, a fim de se evitar perdas e substituições, que descaracterizam as construções.
A NBR 7190 (ASSOCIAÇÃO BRASILEIRA DE NORMAS TÉCNICAS - ABNT, 1997) estabelece que toda estrutura deve ser projetada e construída de modo a satisfazer os requisitos básicos de segurança, permanecendo adequada ao uso previsto e suportando todas as ações durante a sua utilização. Essa norma é recente e posterior à construção de nossos bens históricos, porém, seguramente, seus construtores tinham suas premissas em mente, uma vez que muitas obras, ainda que sem manutenção adequada, permanecem em razoável estado de conservação. Não se pode, entretanto, acreditar que elas continuarão firmes para as futuras gerações, se não receberem ações preventivas ou corretivas.

${ }^{1}$ Universidade Federal de Lavras - Lavras, Minas Gerais, Brazil

Cerne, Lavras, v. 19, n. 3, p. 481-487, jul./set. 2013 
Fundamentadas no uso eficaz de métodos não destrutivos de avaliação de deterioração, na análise das relações das características das deteriorações com as propriedades de resistência da madeira, poderão ser adotadas decisões que contribuam com a preservação e a longevidade do patrimônio em madeira.

A perda de integridade física de estruturas de madeira pode acontecer por diversos fatores, como deformações excessivas ocasionadas pelo excesso de carga ao qual é submetida; formação de rachaduras, em decorrência da retração do material e a deteriorações, principalmente por fungos apodrecedores, propiciada pelo acúmulo de umidade, e por insetos (TELES et al., 2008). Dos agentes deterioradores que atuam na madeira, os bióticos são os de maior importância (CAVALCANTE, 1982), uma vez que a madeira é susceptível a uma variedade de organismos xilófagos. Bactérias podem colonizar e deteriorar madeiras, porém, segundo Belie et al. (2000) e Morrell (2002), os principais agentes xilófagos deterioradores de madeiras são fungos e insetos, cuja ocorrência acontece em função de condições criadas durante a construção do edifício e por falta de manutenção. As propriedades de resistência a esses ataques variam entre diferentes espécies, em uma mesma espécie ou, mesmo, em diferentes partes de uma mesma peça madeira.

De acordo com Feio e Lourenço (2005), as variações de umidade, com consequentes retrações e inchamentos, são responsáveis por tensões internas na madeira, resultando na abertura progressiva de fendas de secagem e no desenvolvimento de distorções diversas. $\mathrm{O}$ efeito de tais fendas na resistência de estruturas varia com sua extensão, profundidade e com a zona da peça e da seção onde ocorrem. Independentemente do eventual desenvolvimento de fendas, os ciclos associados a variações de umidade conduzem, em geral, a uma perda de rigidez das ligações mecânicas, que se traduz no aumento de flexibilidade e de deformações de todo o conjunto estrutural.

De acordo com Råberg et al. (2007) e Ross et al. (1998), a falta de utilização de métodos rápidos e eficazes para detectar e quantificar deteriorações em madeira é um dos fatores que retarda o prognóstico em estruturas. É importante o acesso periódico às condições de integridade da madeira, reparando ou substituindo os membros deteriorados, a fim de se evitarem falhas estruturais (ROSS et al., 2006).

A inspeção visual é o método não destrutivo mais simples de exame, com a qual se verificam sinais externos indicadores de defeitos e ataques. Entretanto, deteriorações internas devem ser avaliadas por outros métodos, como emissão de ondas de tensão e perfurações controladas.

Em termos gerais, de acordo com Ross et al. (2006), uma onda de tensão passa mais rápido por uma madeira sadia do que por uma madeira deteriorada. Medindo-se o tempo de transmissão da onda entre dois transdutores posicionados a uma distância conhecida, a velocidade (v) é determinada, tornando possível estimar o módulo de elasticidade dinâmico, por meio da equação: $\operatorname{MOEd}=\mathrm{v}^{2} * \rho$. Para tanto, é necessário conhecer o valor da densidade $(\rho)$ da madeira, que pode ser estimado com uma amostra retirada da estrutura por um trado ou um formão.

A perfuração controlada é, geralmente, utilizada para confirmar áreas suspeitas de deterioração identificadas por inspeções visuais ou por transmissões de onda de tensão, pois ajuda a definir mais precisamente a extensão e os limites da deterioração. O resistógrafo mede a resistência relativa de um material à perfuração por uma broca de aço em rotação, enquanto ela é introduzida no material a uma velocidade constante. $\mathrm{O}$ aparelho fornece um gráfico que demonstra um perfil da resistência relativa encontrada em cada perfuração realizada.

Um ensaio não destrutivo, geralmente, não danifica a capacidade estrutural da madeira, apresentando outras vantagens como rapidez de execução e relativo baixo custo. Esse tipo de avaliação permite mapear as áreas danificadas em uma estrutura e avaliar sua integridade, auxiliando na decisão sobre a técnica de manutenção ou reabilitação mais indicada (OLIVEIRA et al., 2005).

Neste trabalho, objetivou-se realizar ensaios não destrutivos para inferir sobre a integridade de elementos estruturais de madeira de uma construção histórica de Tiradentes, MG.

\section{MATERIAL E MÉTODOS}

Foram avaliados 1 pilar e 1 viga de madeira, de caráter histórico, situados no Sobrado Ramalho, em Tiradentes, MG. Nesse sobrado, situado à Rua da Câmara, esquina com a Rua Direita, funciona a sede municipal do IPHAN (Instituto do Patrimônio Histórico e Artístico Nacional). Na Figura 1, são mostradas as fachadas frontal e lateral direita (a) e a relação da fachada frontal do edifício, no primeiro plano, com o casario do entorno e a matriz, ao fundo (b).

Nos arquivos do IPHAN, presume-se que a data da construção do sobrado seja de fins do século XVIII ou do início do XIX, momento de consolidação do espaço

Cerne, Lavras, v. 19, n. 3, p. 481-487, jul./set. 2013 


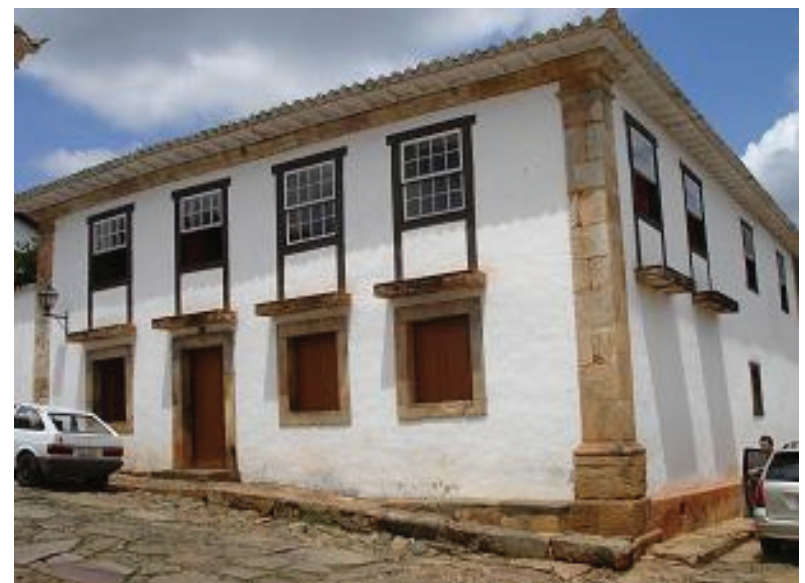

(a)

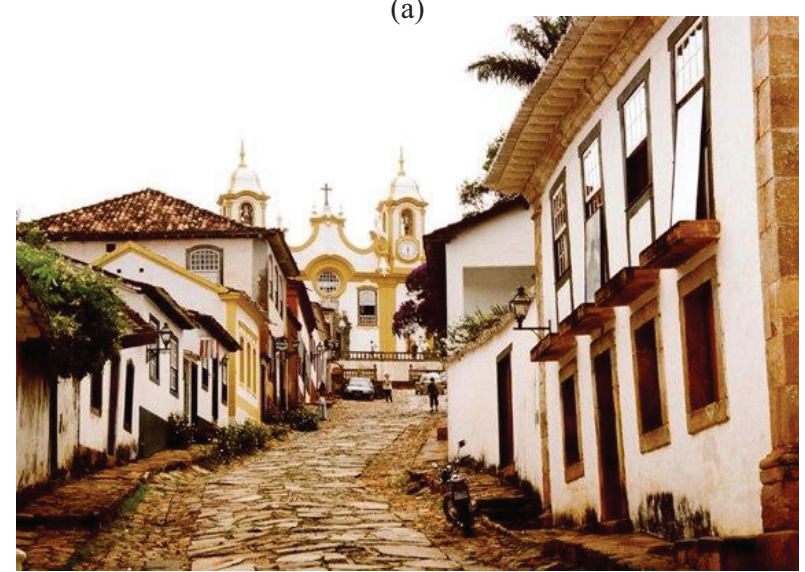

(b)

Figura 1 - Sobrado Ramalho, em que a: fachadas frontal e lateral direita; b: inserção do edifício na Rua da Câmara.

Figure 1 - Sobrado Ramalho, where a: frontal and right side facades; $b$ : insertion of the building at Camara Street.

urbano e de surgimento das construções civis mais sólidas e imponentes. Os vestígios encontrados em seu interior, como o portal decorado em um cômodo sem janelas, caracterizando um oratório particular e a senzala, permitem supor que o sobrado pertenceu a uma família abastada e que foi construído prioritariamente para uso domiciliar. Entretanto, seu primeiro pavimento permitia um uso alternativo e a memória oral da comunidade registra a informação de que ali teria funcionado um posto de recolhimento da tributação colonial, denominado "quinto".

No primeiro pavimento, se encontravam um pilar e uma viga originais da construção, que são o alvo das investigações deste estudo. O pilar apresenta-se continuamente íntegro, aparentemente sem biodeteriorações, e a viga, sem biodeteriorações externas, mas com trincas na face de tração. Na Figura 2, podem ser observados esses elementos estruturais.

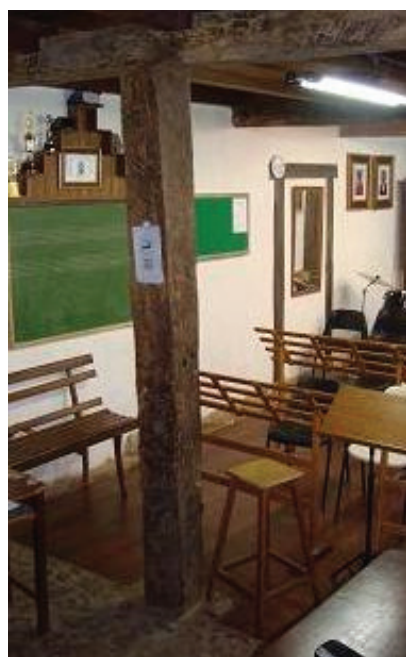

(a)

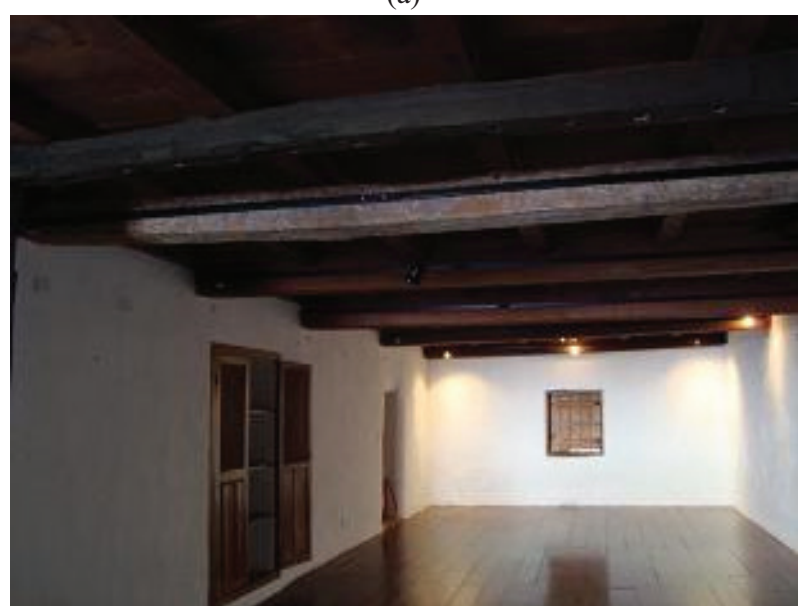

(b)

Figura 2 - Aspecto do pilar e da viga estudados no Sobrado Ramalho.

Figure 2 - Appearance of the pillar and the beam studied in Sobrado Ramalho.

Com amostras retiradas das estruturas por meio de um formão, foram realizadas as análises de densidade, pelo método descrito no item B.6.2 da NBR 7190 (ABNT, 1997). As análises foram feitas no Laboratório de Ciência e Tecnologia da Madeira, da Universidade Federal de Lavras. Como não era objetivo deste trabalho, não foram identificadas as espécies das madeiras das estruturas.

Cerne, Lavras, v. 19, n. 3, p. 481-487, jul./set. 2013 
Para confirmar as suspeitas de deterioração identificadas por inspeção visual, foi utilizado o equipamento stress wave timer, modelo 239A, da fabricante Metriguard. A viga, de $520 \mathrm{~cm}$ de comprimento, foi demarcada com 7 pontos equidistantes, que formaram 6 trechos de aproximadamente $86 \mathrm{~cm}$ cada. Ela foi avaliada nesses trechos, em cada uma das linhas: de compressão (LC), neutra (LN) e de tração (LT), e os módulos de elasticidade dinâmicos dos trechos foram calculados. Na Figura 3, observa-se parte da viga; mais especificamente, observam-se dois dos seis trechos, com o esquema de linhas e pontos, em que, como exemplo, a LC possui os pontos $1 \mathrm{C}, 2 \mathrm{C}$ e $3 \mathrm{C}$. Também podem ser observadas as direções horizontal ( $\mathrm{DH}$ no ponto $1 \mathrm{~N}, \mathrm{DH}$ no ponto $2 \mathrm{~N}$, DH no ponto $3 \mathrm{~N}$ ) e vertical (DV) de aplicação do resistógrafo na viga, que será explicada mais adiante.

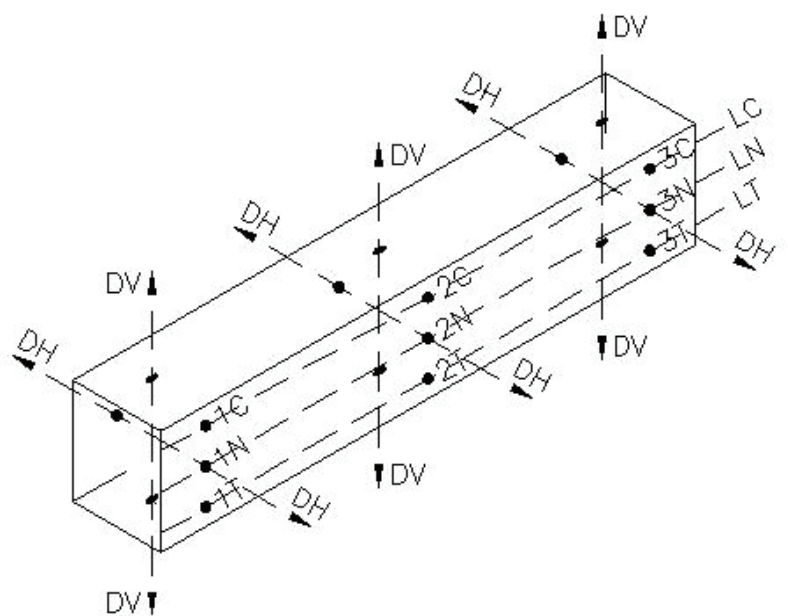

Legenda

(aplicação do stress wave timer):

LC $=$ Linha de compressão;

$\mathrm{LN}=$ Linha neutra;

$\mathrm{LT}=$ Linha de tração.

Legenda

(aplicação do resistógrafo): $\mathrm{DH}=$ direção horizontal; DV $=$ direção vertical.

Figura 3 - Diagrama de direções, linhas e pontos de aplicação dos equipamentos em parte da viga.

Figure 3-Diagram of directions, lines and points of application of the equipments at part of the beam.

Já, no pilar, de $255 \mathrm{~cm}$ de comprimento, o stress wave timer foi utilizado em trechos formados por cinco pontos (por exemplo, 1C, 2C, 3C, 4C e 5C), distantes, aproximadamente, $63 \mathrm{~cm}$ entre si, em 3 alinhamentos diferentes (LC, LN e LT), conforme pode ser observado na Figura 4. Foram avaliados 10 trechos em cada alinhamento: de 1 a 2 ; de 1 a 3 , de 1 a 4 , de 1 a 5 , de

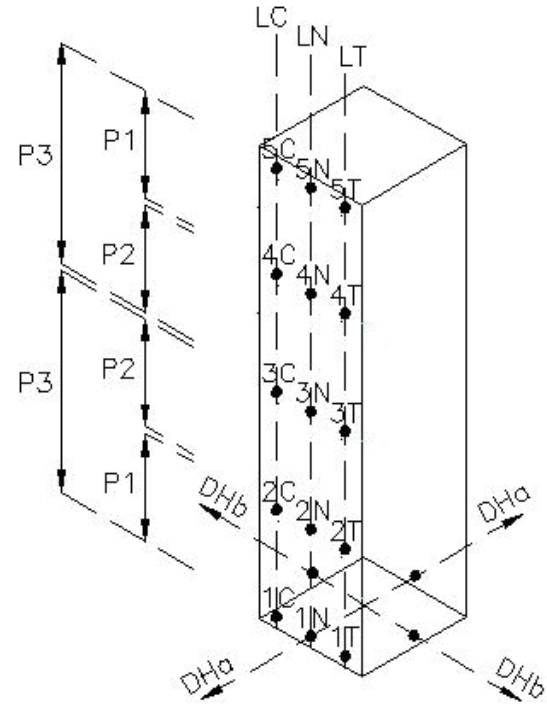

Legenda (aplicação do stress wave timer):

P1: trechos de 1 a 2 e de 5 a 4 , nas LC, LN e LT;

P2: trechos de 2 a 3 e de 4 a 3, nas LC, LN e LT;

P3: trechos de 1 a 3 e de 5 a 3, nas LC, LN e LT;

$\mathrm{LC}=$ Alinhamento $\mathrm{C}$;

$\mathrm{LN}=$ Alinhamento $\mathrm{N}$;

$\mathrm{LT}=$ Alinhamento $\mathrm{T}$.

Legenda (aplicação do resistógrafo):

$\mathrm{DHa}=$ direção horizontal a;

$\mathrm{DHb}=$ direção horizontal $\mathrm{b}$.

Figura 4 - Diagrama de direções, alinhamentos, pontos e posições de aplicação dos equipamentos no pilar.

Figure 4 - Diagram of directions, alignments, points and positions of application of the equipments at the pillar.

2 a 3 , de 2 a 4 , de 2 a 5 , de 3 a 4 , de 3 a 5 e de 4 a 5 . Para possibilitar uma comparação entre os alinhamentos de aplicação do stress wave timer, foi calculada a média dos módulos de elasticidade dos 10 trechos analisados em cada alinhamento.

O resistógrafo, modelo Resistograph ${ }^{\circledR}$ F-400S, do fabricante $I M L$, foi utilizado para perfurar seções com uma broca de aço de $400 \mathrm{~mm}$ de comprimento e $3 \mathrm{~mm}$ de diâmetro. Tanto na viga como no pilar, a princípio, foram realizadas perfurações somente a partir dos pontos centrais (linha neutra LN ou alinhamento LN, respectivamente), na direção DH ou DHa. Em seguida, perfurou-se a mesma seção, porém na direção perpendicular à anterior, denominada DV ou DHb. Assim, foram produzidos gráficos que representam a resistência da madeira à perfuração da broca ao longo de dois caminhos percorridos por ela na mesma seção. Para a análise de variância,

Cerne, Lavras, v. 19, n. 3, p. 481-487, jul./set. 2013 
o modelo admitido foi o inteiramente casualizado, em esquema fatorial com um fator (direção) ou com dois fatores (viga e direção ou pilar e direção). O aplicativo utilizado nas análises foi o SISVAR (FERREIRA, 2000) e o nível máximo de erro admitido nas rejeições das hipóteses foi de $5 \%$.

\section{RESULTADOS E DISCUSSÃO}

A representação gráfica da planta dos ambientes onde se encontram a viga e o pilar pode ser vista na Figura 5.

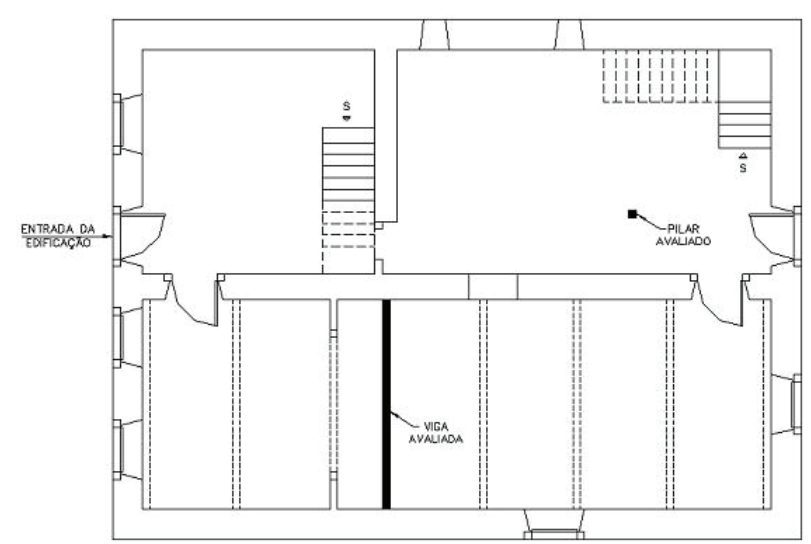

Figura 5 - Representação dos ambientes do primeiro pavimento do Sobrado Ramalho, com destaque para o pilar e a viga avaliados (sem escala).

Figure 5 - Representation of the rooms at the first floor of Sobrado Ramalho, with emphasis on the pillar and the beam evaluated (without scale).

\subsection{Pilar}

As médias dos módulos de elasticidade dos 10 trechos analisados por alinhamento foi calculada para a comparação entre os alinhamentos de aplicação do stress wave timer. Elas demonstraram que não houve variação significativa entre os alinhamentos: LC (10.159 kgf/ $\mathrm{cm}^{2}$ ), LN (10.677 kgf/ $\left.\mathrm{cm}^{2}\right)$, e LT (10.288 kgf/ $\left.\mathrm{cm}^{2}\right)$. Pode-se supor que, pelo fato de o pilar apresentar-se bastante íntegro, sem deteriorações aparentes, de seção praticamente quadrada, e de não sofrer carregamento excêntrico, os esforços que atuam sobre o pilar são distribuídos de maneira uniforme ao longo de seu comprimento, fazendo com que a média dos módulos de elasticidade seja semelhante nos diferentes alinhamentos estudados.
Com relação ao resistógrafo, os dados da Tabela 1, que apresenta o resumo da análise de variância para a variável amplitude, demonstraram que as direções de aplicação do aparelho não diferem entre si $(\mathrm{P}<0,3422)$. Conforme afirmado anteriormente, pode-se supor que os esforços que atuam sobre ele sejam distribuídos de maneira uniforme, não se diferenciando os perfis das seções nas direções DHa e DHb.

Tabela 1 - Resumo da análise de variância para amplitude fornecida pelo resistógrafo no pilar.

Table 1 - Summary of ANOVA for amplitude provided by the resistograph on the pillar.

\begin{tabular}{lccc}
\hline FV & GL & Quadrados Médios & Pr $>\mathrm{Fc}$ \\
\hline Direção & 1 & 111,82 & 0,3422 \\
Erro & 8 & 109,67 & \\
\hline Total & 9 & & \\
\hline CV & & $31,96 \%$ & \\
\hline
\end{tabular}

\subsection{Viga}

O stress wave timer não pôde ser aplicado na linha neutra, em razão da presença de uma canaleta metálica de energia elétrica ao longo do comprimento da viga, localizado na região central de sua altura. É importante ressaltar que essa viga apresentava trincas intermediáriocentrais na linha de tração, destacadas na Figura 6, enfatizando-se, assim, a importância de se estudar as linhas separadamente.

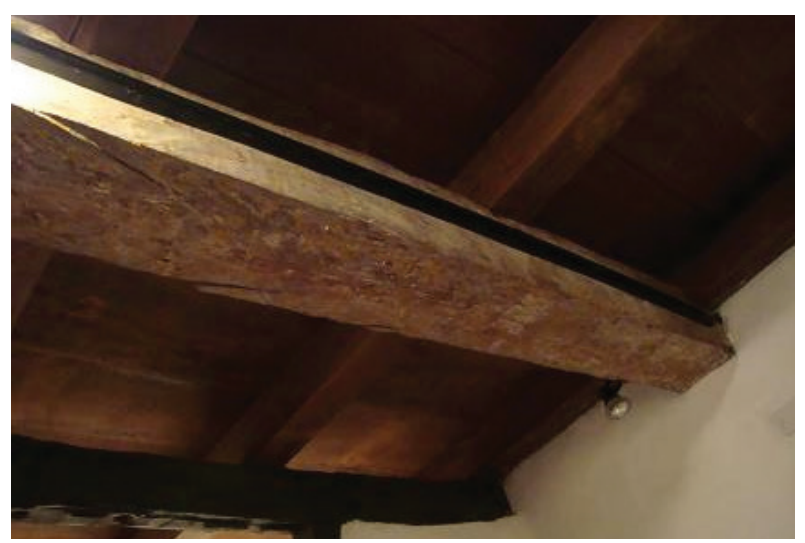

Figura 6 - Aspectos das rupturas da viga avaliada no Sobrado Ramalho.

Figure 6-Aspects of the cracks in the evaluated beam at Sobrado Ramalho.

Cerne, Lavras, v. 19, n. 3, p. 481-487, jul./set. 2013 
As médias obtidas entre os módulos de elasticidade dinâmicos calculados de todos os trechos avaliados por linha mostraram que as linhas de compressão e de tração não apresentaram diferenças significativas entre si (12.746 kgf $/ \mathrm{cm}^{2}$ e $13.263 \mathrm{kgf} / \mathrm{cm}^{2}$, respectivamente).

Para possibilitar uma análise gráfica de todos os trechos da viga, foram utilizados os valores de módulo de elasticidade dos trechos de 1 a 2 , de 2 a 3 , de 3 a 4, de 4 a 5 , de 5 a 6 e de 6 a 7 , para as duas linhas. Os resultados são apresentados nas Figuras 7 e 8.

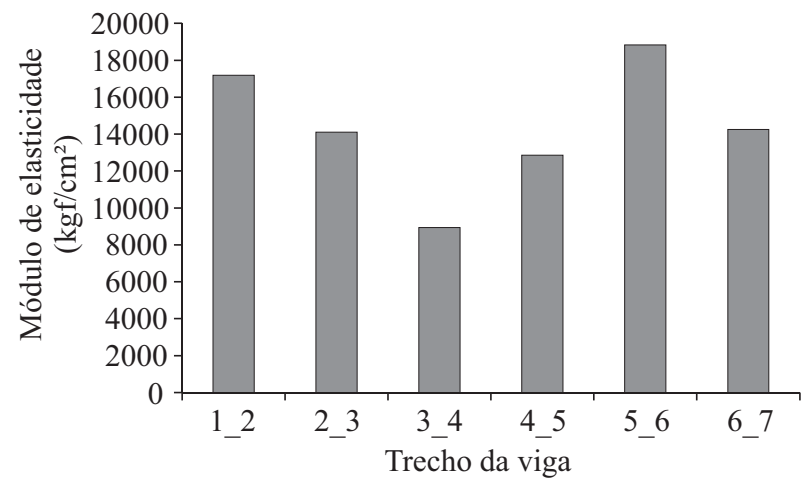

Figura 7 - Módulo de elasticidade em trechos ao longo da viga, na linha de compressão.

Figure 7 -Modulus of elasticity in patches along the beam, in the compression line.

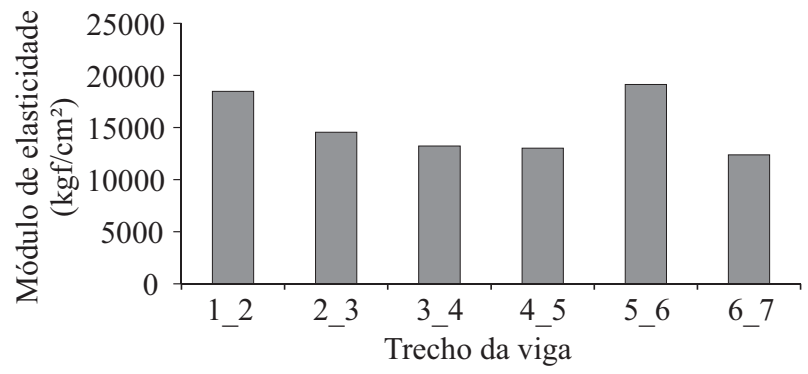

Figura 8 - Módulo de elasticidade em trechos ao longo da viga, na linha de tração.

Figure 8 -Modulus of elasticity in patches along the beam, in the traction line.

Tanto na linha de compressão como na linha de tração, os dois trechos dos extremos apresentaram valores de módulos maiores e semelhantes, enquanto os dois trechos centrais apresentaram menores valores. Ainda segundo os dados das Figuras 7 e 8, observou-se que, ao longo da linha de compressão, os valores de módulos de elasticidade nos trechos centrais variaram mais entre si, embora na linha de tração não tenha havido variação, apesar da existência das trincas nessa última.

Com relação à aplicação do resistógrafo na viga, na Tabela 2, onde se apresenta o resumo da análise de variância para a variável amplitude, demonstra que as direções de aplicação do aparelho diferem entre si $(\mathrm{P}<0,0353)$.

Tabela 2 - Resumo da análise de variância para amplitude fornecida pelo resistógrafo na viga.

Table 2 - Summary of ANOVA for amplitude provided by the resistograph on the beam.

\begin{tabular}{lccc}
\hline FV & GL & Quadrados Médios & $\operatorname{Pr}>\mathrm{Fc}$ \\
\hline Direção & 1 & 935 & 0,0353 \\
Erro & 11 & 163 & \\
\hline Total & 12 & & \\
\hline CV & & $33,82 \%$ & \\
\hline
\end{tabular}

Pelos resultados do teste de Tukey $(\mathrm{P}<0,05)$, apresentados na Tabela 3, é demonstrada a diferença entre as médias das duas direções.

Tabela 3 - Médias de amplitudes das direções de aplicação do resistógrafo na viga.

Table 3 -Means of amplitudes of the directions of applications of the resistograph on the beam.

\begin{tabular}{lc}
\hline Direção & Média das amplitudes (\%) \\
\hline DH (horizontal) & $28,55 \mathrm{a}$ \\
DV (vertical) & $45,56 \mathrm{~b}$ \\
\hline
\end{tabular}

Na direção horizontal (DH) de aplicação do resistógrafo, foram perfurados os pontos da viga na linha de tração, uma vez que a linha neutra estava inacessível pelo trilho de iluminação. Na direção vertical (DV), foram perfurados os pontos na direção perpendicular à anterior, ou seja, atravessando as regiões tracionadas e comprimidas da seção transversal da viga. O teste de Tukey mostrou que a média de amplitudes na direção DV foi maior que na DH. Esse fato demonstrou que a broca, quando passou pelos três planos (DV), encontrou mais resistência do que quando passou apenas pela linha de tração $(\mathrm{DH})$. Essa diferença de resistência se deve ao fato de a broca, na direção DH, ter perfurado a viga passando pelas trincas, que formaram lascas separadas da viga, deixando espaços vazios entre elas (Figura 6). Na direção DV, o resistógrafo perfurou a viga internamente, de baixo para cima, por onde não haviam vazios aparentes, o que dificulta a penetração da broca.

Cerne, Lavras, v. 19, n. 3, p. 481-487, jul./set. 2013 
$\mathrm{O}$ ambiente onde se encontra a viga avaliada possui outras vigas, não originais, que ajudam a suportar as cargas do piso superior. Entretanto, nenhuma delas recebe a carga de uma parede, como é o caso da viga em questão. Apesar disso, pela qualidade de sua madeira e à sua robustez, juntamente à fartura de vigas, opção estrutural comum em edifícios históricos, acredita-se que a integridade da viga esteja garantida. Sugere-se uma avaliação estrutural de acordo com as normas vigentes.

\section{CONCLUSÕES}

Quando da aplicação do equipamento stress wave timer, as estruturas avaliadas apresentaram módulos de elasticidade dinâmicos estatisticamente iguais nos alinhamentos estudados, o que demonstra a homogeneidade de cada peça.

Quanto ao resistógrafo, quando aplicado no pilar, não diferiram significativamente as direções de aplicação do aparelho, o que corrobora com a avaliação visual prévia, que o classificou como "íntegro". Já, nos ensaios da viga, houve diferenças significativas em função da direção de aplicação, demonstrando que a peça não apresenta uniformidade, o que também se esperava, em razão da presença das trincas em determinadas seções avaliadas.

A presença de trincas na viga não afetou sua capacidade elástica, ainda que tenham ocorrido pequenas reduções de seção. Sugere-se uma avaliação estrutural de acordo com as normas vigentes.

Os dados gerados pelos equipamentos utilizados nesse trabalho podem ajudar a embasar decisões sobre a substituição integral de elementos estruturais de madeira, que vem ocorrendo com frequência no patrimônio histórico brasileiro.

\section{REFERÊNCIAS}

ASSOCIAÇÃO BRASILEIRA DE NORMAS TÉCNICAS. NBR-7190: projeto de estruturas de madeira. Rio de Janeiro, 1997. $107 \mathrm{p}$.

BELIE, N. de; RICHARDSON, M.; BRAAM, C. R.; SVENNERSTEDT, B.; LENEHAN, J. J.; SONCK, B. Durability of building materials and components in the agricultural environment: part I, the agricultural environment and timber structures. Journal of Agricultural Engineering Research, London, v. 75, n. 3, p. 225-241, Mar. 2000.
CAVALCANTE, M. S. Deterioração biológica e preservação de madeiras. São Paulo: Instituto de Pesquisas Tecnológicas do Estado de São Paulo, 1982. 41 p. (Pesquisa \& Desenvolvimento, 8).

FEIO, A. O.; LOURENÇO, P. B. Possibilidades e aplicações de ensaios não destrutivos. In: ENCONTRO SOBRE A MADEIRA E SUAS APLICAÇÕES NOBRES "BEM UTILIZAR A MADEIRA”, 1., 2005, Lisboa. Anais... Lisboa: ISISE, 2005. 1 CD-ROM.

FERREIRA, D. F. Análises estatísticas por meio do Sisvar para Windows versão 4.0. In: REUNIÃO ANUAL DA REGIÃO BRASILEIRA DA SOCIEDADE INTERNACIONAL DE BIOMETRIA, 45., 2000, São Carlos. Anais... São Carlos: UFSCar, 2000. p. 255-258.

MORRELL, J. J. Based building components: what have we learned? International Biodeterioration \& Biodegradation, Birmingham, v. 49, n. 4, p. 253-258, June 2002.

OLIVEIRA, F. G. R.; CANDIANB, M.; LUCCHETTEB, F. F.; SALGONB, J. L.; SALESB, A. A technical note on the relationship between ultrasonic velocity and moisture content of Brazilian hardwood (Goupia glabra). Building and Environment, Oxford, v. 40, n. 2, p. 297-300, Feb. 2005.

RÅBERG, U.; TERZIEV, N.; LAND, C. J. Early soft rot colonization of Scots sapwood pine in above-ground exposure. International Biodeterioration \& Biodegradation, Birmingham, v. 63, n. 2, p. 236-240, Mar. 2007.

ROSS, R. J.; BRASHAW, B. K.; PELLERIN, R. F. Nondestructive evaluation of wood. Forest Products Journal, Madison, v. 48, n. 1, p. 14-19, Jan. 1998.

ROSS, R. J.; BRASHAW, B. K.; WANG, X. Structural condition assessment of in-service wood. Forest Products Journal, Madison, v. 56, n. 6, p. 4-8, June 2006.

TELES, R. F.; RIBEIRO, P. G.; DEL MENEZZI, C. H. S. Avaliação estrutural não-destrutiva do prédio Oca II, Universidade de Brasília. In: ENCONTRO BRASILEIRO EM MADEIRAS E EM ESTRUTURAS DE MADEIRA, 11., 2008, Londrina. Anais... Londrina: EBRAMEM, 2008. 1 CD-ROM.

Recebido: 2 de junho de 2010; aceito: 25 de março de 2013.

Cerne, Lavras, v. 19, n. 3, p. 481-487, jul./set. 2013 
